\title{
Constraints on the meta-galactic hydrogen ionisation rate from the Lyman- $\alpha$ forest opacity
}

\author{
James S. Bolton ${ }^{1}$, Martin G. Haehnelt ${ }^{1}$, Matteo Viel ${ }^{1}$, \\ and Volker Springel ${ }^{2}$ \\ ${ }^{1}$ Institute of Astronomy, University of Cambridge, Madingley Road, \\ Cambridge, CB3 0HA, UK \\ ${ }^{2}$ Max-Planck-Institut für Astrophysik, Karl-Schwarzschild-Str. 1, \\ Garching bei München, Germany
}

\begin{abstract}
Understanding the sources responsible for reionizing the Universe is a key goal of observational cosmology. A discrepancy has existed between the meta-galactic hydrogen ionisation rate, $\Gamma_{\mathrm{HI}}$, predicted by early hydrodynamical simulations of the Lyman- $\alpha$ forest if scaled to appropriate assumptions for the IGM temperature, when compared to values predicted from the proximity effect. We present new estimates for $\Gamma_{\mathrm{HI}}$ in the redshift range $2<z<4$ based on hydrodynamical simulations of the Lyman- $\alpha$ forest opacity. Within the current concordance cosmology, and assuming updated QSO emissivity rates, a substantial contribution to the UV background from young star-forming galaxies appears to be required over the entire redshift range. Our results are consistent with lower-end estimates from the proximity effect. It is also found that the errors on the ionisation rate are dominated by uncertainties in the thermal state of the intergalactic medium and the r.m.s fluctuation amplitude at the Jeans scale.
\end{abstract}

\section{Introduction}

The series of low column density $\left(10^{12.5}<N_{\mathrm{HI}}<10^{14.5} \mathrm{~cm}^{-2}\right)$ Ly $\alpha$ absorption features seen blue-ward of the Ly $\alpha$ emission line in high redshift quasi-stellar object (QSO) spectra trace the distribution of neutral hydrogen in the intergalactic medium (IGM). This forest of absorption lines provides a unique, unbiased probe of the thermal and ionisation history of the IGM along the QSO line-of-sight. This has motivated many authors to use hydrodynamical simulations of structure formation, calibrated to reproduce the observed properties of the forest obtained from high resolution QSO spectra, to infer the amplitude of the meta-galactic UV background (see the seminal paper by Rauch et al. 1997, and most recently Tytler et al. 2004). Consequently there are a wide range of estimates for the hydrogen ionisation rate per atom, $\Gamma_{\mathrm{HI}}$, inferred from the Ly $\alpha$ forest opacity using numerical simulations with different assumptions. This complicates the comparison with determinations of the ionisation rate from estimates of the integrated emission from observed QSOs and/or galaxies (e.g. Haardt \& Madau 1996, hereafter HM96; Madau, Haardt \& Rees 1999) and estimates using the proximity effect (e.g. Scott et al. 2000) which both also have rather large uncertainties. Furthermore, Steidel et al. (2001), in a study of Lyman break galaxies at $z \simeq 3.4$, suggested that the intensity of the ionising background may be a factor of a few larger than in the model of HM96 due to a large contribution from star-forming galaxies.

We discuss new estimates of the meta-galactic hydrogen ionisation rate presented in Bolton et al. (2005), inferred from state-of-the-art hydrodynamical simulations of the 
Ly $\alpha$ forest. These estimates are consistent with a substantial contribution from galaxies to the amplitude of the UV background at the hydrogen ionisation edge, and are in agreement with other observational estimates of the dimensionless quantity $\Gamma_{-12}=$ $\Gamma_{\mathrm{HI}} / 10^{-12} \mathrm{~s}^{-1}$.

\section{Numerical code and convergence}

We use a suite of 21 high resolution hydrodynamical simulations run using a new version of GADGET (Springel, Yoshida \& White, 2001) to investigate in detail the dependence of $\Gamma_{\mathrm{HI}}$ on physical and numerical parameters (for a detailed description of the simulations see Bolton et al. 2005). The cosmological parameters for our adopted fiducial model are:

$$
\left(\Omega_{\mathrm{m}}, \Omega_{\mathrm{b}} h^{2}, h, \sigma_{8}\right)=(0.26 \pm 0.04,0.024 \pm 0.001,0.72 \pm 0.04,0.85 \pm 0.05),
$$

consistent with the results of Spergel et al. (2003), assuming a flat, vacuum energy dominated cosmological model. We assume $n=0.95$ and a helium mass fraction of $Y=0.24$. In addition, we adopt fiducial values for the thermal state of the gas at mean cosmic density; $T_{0}=[11200,17800,12500] \pm 5000 \mathrm{~K}$ at $z=[2,3,4]$ and $\gamma=1.3 \pm 0.3$ for the index of the gas effective equation of state (Hui \& Gnedin 1997), based on the results of Schaye et al. (2000). We linearly rescale the opacity of the artificial Ly $\alpha$ forest spectra we construct to match the central values for the Ly $\alpha$ forest effective optical depth taken from the fitting formula of Schaye et al. (2003): $\tau_{\text {eff }}=[0.130 \pm 0.021,0.362 \pm 0.036,0.805 \pm 0.070]$. We treat the amplitude of the UV background as a free parameter, so rescaling the neutral hydrogen opacity corresponds to linearly rescaling the hydrogen ionisation rate by the same amount.

We initially ran 7 simulations of our fiducial model with differing box size and resolution to assess the degree of numerical convergence. We found that simulations with less than $400^{3}$ gas particles within a $30 h^{-1}$ co-moving Mpc box do not achieve adequate numerical convergence. We take our fiducial model to have $200^{3}$ gas particles within a $15 h^{-1}$ comoving Mpc box; we estimate the value of $\Gamma_{-12}$ we infer will be systematically higher by around 8 per cent due to the combined error from box size and resolution.

\section{Scaling relations for $\Gamma_{-12}$ from simulated absorption spectra}

When inferring $\Gamma_{-12}$ from hydrodynamical simulations and subsequently rescaling with different parameters, it is generally assumed that (e.g. Rauch et al. 1997):

$$
\Gamma_{-12} \propto \Omega_{\mathrm{b}}^{2} h^{3} T^{-0.7} \Omega_{\mathrm{m}}^{-0.5} .
$$

It is implicit in this relation that the density and velocity distribution, along with the effective equation of state of the Ly $\alpha$ absorbers, remain unchanged for different values of $\Omega_{\mathrm{b}}, h, \Omega_{\mathrm{m}}, T$ and $\Gamma_{-12}$. We tested the validity of Eq. 3.1 using 14 simulations in addition to our fiducial model, by varying the parameters $T, \Omega_{\mathrm{m}}$, and $\sigma_{8}$ in each run. We also rescale to different values for the temperature-density relation index $\gamma$ and the effective optical depth in post-processing. We do not discuss the scaling with $\Omega_{\mathrm{b}}$ and $h$, since our analysis revealed that Eq. 3.1 holds extremely well for these parameters. Figs. 1 and 2 and Table 1 summarise the main results of this study. We find that $\Gamma_{-12}$ scales around our fiducial model as:

$$
\Gamma_{-12} \propto \Omega_{\mathrm{b}}^{2} h^{3} T^{x_{1}(z)} \gamma^{x_{2}(z)} \Omega_{\mathrm{m}}^{x_{3}(z)} \sigma_{8}^{x_{4}(z)} \tau_{\mathrm{eff}}^{x_{5}(z)},
$$



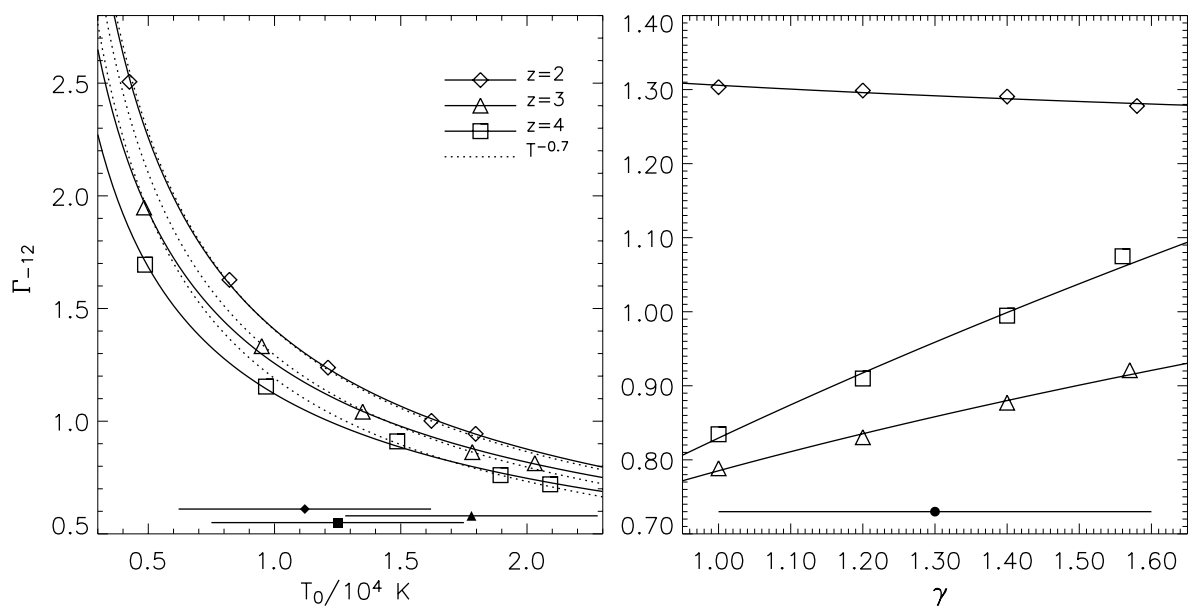

Figure 1. Left: the dependence of $\Gamma_{-12}$ on the gas temperature at mean density $T_{0}$ at the three different redshifts indicated on the plot. Solid curves show a least squares fit and the dotted curves shows the $T^{-0.7}$ scaling due to the temperature dependence of the recombination coefficient. The filled symbols show the fiducial temperatures and their uncertainties. Right: the dependence of $\Gamma_{-12}$ on the index of the temperature density relation $\gamma$. The filled circle shows our fiducial value of $\gamma$ and its uncertainty.

Table 1. The redshift dependent indices from our scaling relation of $\Gamma_{-12}$ with several cosmological and astrophysical parameters.

\begin{tabular}{cccccc}
\hline & $T$ & $\gamma$ & $\Omega_{\mathrm{m}}$ & $\sigma_{8}$ & $\tau_{\text {eff }}$ \\
\hline$z$ & $x_{1}(z)$ & $x_{2}(z)$ & $x_{3}(z)$ & $x_{4}(z)$ & $x_{5}(z)$ \\
\hline 2 & -0.68 & -0.04 & -1.00 & -0.90 & -1.44 \\
3 & -0.62 & 0.34 & -1.04 & -0.99 & -1.61 \\
4 & -0.59 & 0.55 & -1.16 & -1.26 & -1.68 \\
\hline
\end{tabular}

where we tabulate the indices of Eq. 3.2 in Table 1. We stress that this scaling relation is likely to be somewhat model dependent and should not be applied to models with parameters very different from our fiducial model without further checks. However, it does provide a clear picture of the degeneracies which exist between $\Gamma_{-12}$ and other parameters within our simulations, and is independent of assumptions about the gas distribution, effective equation of state, and ionised gas fraction of the low density IGM. In particular, the $\operatorname{Ly} \alpha$ optical depth at fixed r.m.s. fluctuation amplitude $\sigma_{8}$ is more strongly dependent on $\Omega_{\mathrm{m}}$ than Eq. 3.1 suggests due to changes in the gas density distribution. Thermal broadening also produces a deviation from the scaling of Eq. 3.1 for temperature, although this change is much less dramatic. Consequently, we urge caution when using Eq. 3.1, especially if comparing models with differing $\Omega_{\mathrm{m}}$. We also find a strong dependence of $\Gamma_{-12}$ on the effective optical depth of the Ly $\alpha$ forest which the simulated spectra are scaled to match.

\section{Results}

Using our scaling relations, we estimated the error on the values of $\Gamma_{-12}$ from our fiducial simulation using the uncertainties on our fiducial parameter values taken from 

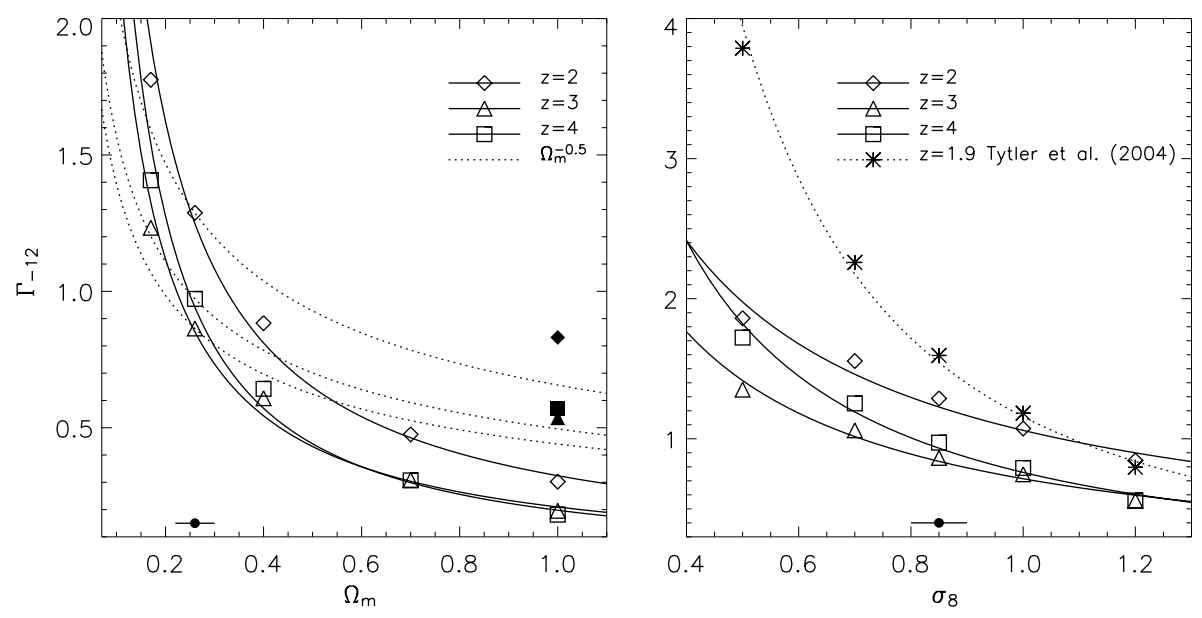

Figure 2. Left: the dependence of the estimated $\Gamma_{-12}$ on $\Omega_{\mathrm{m}}$ for the three different redshifts indicated on the plot. The solid curves are a least-square fit. The dotted lines show the $\Omega_{\mathrm{m}}^{-0.5}$ scaling. Filled points are obtained for a model with $\Omega_{\mathrm{m}}=1.0$ and the the same r.m.s. fluctuation amplitude at a scale of $30 \mathrm{kms}^{-1}$ as our fiducial model. The filled circle shows our fiducial value of $\Omega_{\mathrm{m}}=0.26$ and its uncertainty. Right: the dependence of $\Gamma_{-12}$ on $\sigma_{8}$. The dotted curve shows the result of Tytler et al. (2004) at $z=1.9$, assuming $\langle F\rangle_{\mathrm{obs}}=0.882 \pm 0.01$. The filled circle shows our fiducial value of $\sigma_{8}$ and its uncertainty

the literature. The error budget is listed in Table 2. We find the largest contribution is from the uncertainty in the gas temperature. There is also a large contribution from the uncertainty in the effective optical depth, especially at $z=2$. Interestingly, the uncertainty in $\Omega_{\mathrm{m}}$ also gives a substantial contribution to the total error budget. This is primarily due to the sensitivity of $\Gamma_{-12}$ on $\Omega_{\mathrm{m}}$ due to changes in the r.m.s. fluctuations of the gas density at the Jeans scale. Other uncertainties are less important, in particular $\Omega_{\mathrm{b}} h^{2}, \sigma_{8}$, and $h$. Using our fiducial model, we find the Ly $\alpha$ effective optical depth of the IGM at $z=[2,3,4]$ is reproduced by $\Gamma_{-12}=\left[1.29 \pm_{0.46}^{0.80}, 0.86 \pm_{0.26}^{0.34}, 0.97 \pm_{0.33}^{0.48}\right]$.

We compare our results with other observational estimates of $\Gamma_{-12}$ in Fig. 3 . The filled triangles show the meta-galactic hydrogen ionisation rates computed from the updated UV background model of Madau, Haardt \& Rees (1999), hereafter MHR99 (Francesco Haardt, private communication), based on the contribution from QSOs and re-processing by the IGM (e.g. HM96). Our data appear to be inconsistent with the IGM being kept ionised by QSOs at $z \leqslant 4$. For comparison, the filled squares give $\Gamma_{-12}$ calculated using the QSO rate above, plus an additional source in the form of Young Star Forming Galaxies (YSFGs). These ionisation rates are in good agreement with our results, suggesting that a substantial contribution from galaxies appears to be required at all redshifts.

Constraints on $\Gamma_{-12}$ from the proximity effect measurements of Scott et al. (2000) are shown by the hatched box. Our results are consistent with the lower end of these estimates. The filled circle gives the $\Gamma_{-12}$ we calculate assuming a spectral index of $\alpha=1.8$ using the meta-galactic ionising radiation intensity inferred from Lyman-break galaxies (Steidel et al. 2001). Finally we also plot the value of $\Gamma_{-12}$ inferred by Tytler et $a l$. in their study of the Ly $\alpha$ forest at $z=1.9$. The error bars include the uncertainties in $\tau_{\text {eff }}, \sigma_{8}$, and $\Omega_{\mathrm{b}}$, based on scaling from their hydrodynamical simulations. Our data is in reasonable agreement with the Tytler et al. (2004) result, allowing for differences between the exact gas temperatures and numerical method. 
Table 2. Percentage error budget for $\Gamma_{-12}$ from estimates of various cosmological and astrophysical parameters, listed approximately in order of importance. The total error is obtained by adding the individual errors in quadrature.

\begin{tabular}{cccc}
\hline Parameter & $z=2.00$ & $z=3.00$ & $z=4.00$ \\
\hline$T$ & ${ }_{-22}^{+50}$ & ${ }_{-14}^{+23}$ & ${ }_{-18}^{+35}$ \\
$\Omega_{\mathrm{m}}$ & ${ }_{-13}^{+18}$ & ${ }_{-14}^{+19}$ & ${ }_{-15}^{+21}$ \\
$\tau_{\text {eff }}$ & ${ }_{-19}^{+29}$ & ${ }_{-14}^{+18}$ & ${ }_{-13}^{+17}$ \\
Numerical & \pm 10 & \pm 10 & \pm 10 \\
$\gamma$ & \pm 1 & ${ }_{-9}^{+7}$ & ${ }_{-13}^{+12}$ \\
$\Omega_{\mathrm{b}} h^{2}$ & ${ }_{-8}^{+9}$ & -9 & ${ }_{-8}^{+9}$ \\
$\sigma_{8}$ & ${ }_{-5}^{+6}$ & \pm 6 & ${ }_{-7}^{+8}$ \\
$h$ & \pm 6 & \pm 6 & \pm 6 \\
\hline Total & ${ }_{-36}^{+62}$ & ${ }_{-30}^{+39}$ & ${ }_{-34}^{+49}$ \\
\hline
\end{tabular}

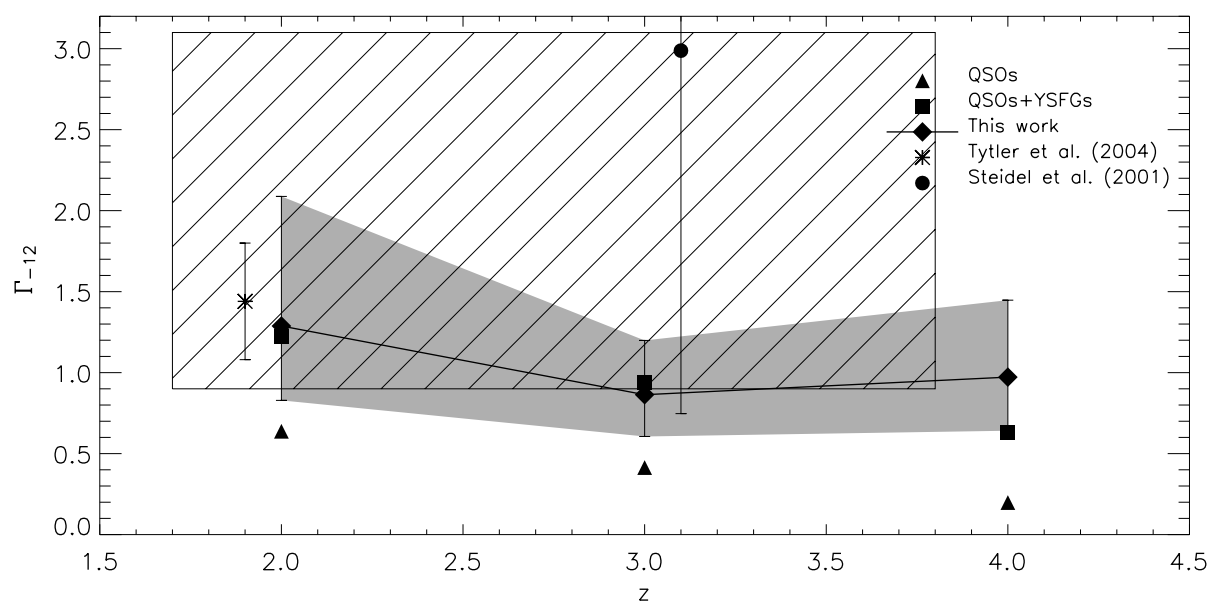

Figure 3. Comparison of our best estimate for $\Gamma_{-12}$ at $z=[2,3,4]$ with constraints from observations. Our data are plotted with filled diamonds, and the grey shaded area shows the error bounds. The filled squares and triangles show the estimated contribution to the meta-galactic ionisation rate from QSOs+galaxies and QSOs alone, based on estimates from the updated model of Madau, Haardt \& Rees (1999) including UV photons from re-processing by the IGM. The hatched box gives the constraint on $\Gamma_{-12}$ from the proximity effect (Scott et al. 2000) and the estimate from Lyman-break galaxies assuming a global spectral index of $\alpha=1.8$ is plotted with a filled circle (Steidel et al. 2001). The data point has been offset from $z=3$ for clarity, and the upper error limit is not shown. The star shows the best estimate of $\Gamma_{-12}$ at $z=1.9$ from Tytler et al. (2004), including their errors from the uncertainty in $\tau_{\text {eff }}, \sigma_{8}$, and $\Omega_{\mathrm{b}}$.

\section{Conclusions}

In recent years, compelling observational evidence has led to the acceptance of a standard cosmological model which is flat, has low matter density, and a substantial contribution of vacuum energy to the total energy density. Within this 'concordance' cosmological model the current generation of hydrodynamical simulations predict values for the metagalactic hydrogen ionisation rate, required to reproduce the effective Ly $\alpha$ optical depth of the IGM in the range $z=2-4$, which are about a factor of four larger than those in an Einstein-de Sitter model with the same r.m.s. density fluctuation amplitude $\sigma_{8}$. 
The ionisation rates estimated from the $\operatorname{Ly} \alpha$ forest opacity are more than a factor of two larger than estimates from the integrated flux of optically/UV bright observed QSOs alone. This discrepancy increases with increasing redshift. We confirm the findings of Tytler et al. (2004) at $z \sim 1.9$ that the estimated ionisation rates from simulations of a $\Lambda$ CDM concordance model are in reasonable agreement with the estimates of the integrated ionising flux from observed QSOs plus a significant contribution from galaxies as in the model of MHR99. The estimates of the ionisation rate are also in agreement with the lower end of the range of values from the proximity effect. This new agreement appears to be in part due to the currently favoured low value of $\Omega_{\mathrm{m}}$, which increases the required value of $\Gamma_{\mathrm{HI}}$, and more accurate measurements of the Ly $\alpha$ forest opacity. We also find that the uncertainty on the magnitude of $\Gamma_{\mathrm{HI}}$ inferred from simulations is greater than previously estimated, with the primary contribution to this error coming from the temperature of the low density gas in the IGM. Better constraints on the thermal history of the IGM are required. Additional physics such as radiative transfer, galactic feedback, and metal enrichment may need to be incorporated into simulations in a more realistic fashion. However, our estimate of the error on $\Gamma_{-12}$ most likely accounts for the modest changes expected from these processes, suggesting we have obtained a consistent constraint on the meta-galactic hydrogen ionisation rate.

\section{Acknowledgements}

We are grateful to Francesco Haardt for making his updated UV background model available to us. The simulations were run using the Altix 3700 supercomputer COSMOS at the Department of Applied Mathematics and Theoretical Physics in Cambridge. COSMOS is a UK-CCC facility which is supported by HEFCE and PPARC. We acknowledge support from the European Community Research and Training Network "The Physics of the Intergalactic Medium", NSF Grant No. PHY99-07949, and PPARC. JSB and MV also thank the IAU for financial assistance.

\section{References}

Bolton, J. S., Haehnelt, M. G., Viel, M., Springel, V., 2005, MNRAS, 357, 1178

Haardt, F., Madau, P., 1996, ApJ, 461, 20

Hui, L., Gnedin, N., 1997, MNRAS, 292, 27

Madau, P., Haardt, F., Rees, M. J., 1999, ApJ, 585, 34

Rauch, M., et al., 1997, ApJ, 489, 7

Schaye, J., Aguirre, A., Kim, T.-S., Theuns, T., Rauch, M., Sargent W. L. W., 2003, ApJ, 596, 768

Schaye, J., Theuns, T., Rauch, M., Efstathiou, G., Sargent W. L. W., 2000, MNRAS, 318, 817

Scott, J., Bechtold, J., Dobrzycki, A., Kulkarni, V. P., 2000, ApJS, 130, 67

Spergel, D. N., et al., 2000, ApJS, 148, 175

Springel, V., Yoshida, N., White, S. D. M., 2001, New Astron., 6, 79

Steidel, C. C., Pettini, M., Adelberger K. L., 2001, ApJ, 546, 665

Tytler, D., et al., 2004, ApJ, 617, 1 